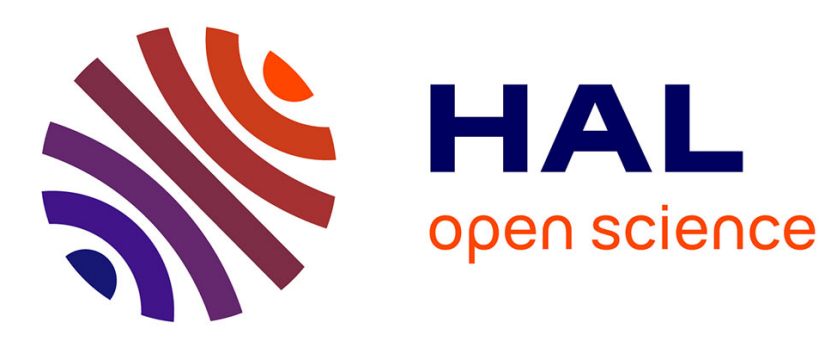

\title{
Monocular Human Shape and Pose with Dense Mesh-borne Local Image Features
}

Shubhendu Jena, Franck Multon, Adnane Boukhayma

\section{To cite this version:}

Shubhendu Jena, Franck Multon, Adnane Boukhayma. Monocular Human Shape and Pose with Dense Mesh-borne Local Image Features. FG 2021 - IEEE International Conference on Automatic Face and Gesture Recognition, Dec 2021, Jodhpur (online), India. pp.1-5, 10.1109/FG52635.2021.9666993 . hal-03524051

\section{HAL Id: hal-03524051 \\ https://hal.inria.fr/hal-03524051}

Submitted on 13 Jan 2022

HAL is a multi-disciplinary open access archive for the deposit and dissemination of scientific research documents, whether they are published or not. The documents may come from teaching and research institutions in France or abroad, or from public or private research centers.
L'archive ouverte pluridisciplinaire HAL, est destinée au dépôt et à la diffusion de documents scientifiques de niveau recherche, publiés ou non, émanant des établissements d'enseignement et de recherche français ou étrangers, des laboratoires publics ou privés. 


\title{
Monocular Human Shape and Pose with Dense Mesh-borne Local Image Features
}

\author{
Shubhendu Jena, Franck Multon, Adnane Boukhayma \\ Inria, Univ. Rennes, CNRS, IRISA, M2S, France
}

\begin{abstract}
We propose to improve on graph convolution based approaches for human shape and pose estimation from monocular input, using pixel-aligned local image features. Given a single input color image, existing graph convolutional network (GCN) based techniques for human shape and pose estimation (e.g. [19]) use a single convolutional neural network (CNN) generated global image feature appended to all mesh vertices equally to initialize the GCN stage, which transforms a template T-posed mesh into the target pose. In contrast, we propose for the first time the idea of using local image features per vertex. These features are sampled from the $\mathrm{CNN}$ image feature maps by utilizing pixel-to-mesh correspondences generated with DensePose [11]. Our quantitative and qualitative results on standard benchmarks show that using local features improves on global ones and leads to competitive performances with respect to the state-of-the-art.
\end{abstract}

\section{INTRODUCTION}

Reconstructing human bodies e.g. [25], [22] and their parts (faces e.g. [7], [1], hands e.g. [4], [9]) from minimal, partial and noisy inputs is one of the most sought-after goals of human centered machine learning. In this regard, human shape and pose recovery from a single color image is a popular problem in computer vision and graphics spurring a vast research literature, with applications in various areas such as action recognition, avatarization, human machine interaction, etc. While earlier approaches to 3D reconstruction relied on multi-view triangulation or depth information, the recent surge of deep learning has allowed the reduction of acquisition constraints to as little as a single input RGB image. The ill-posedness of this monocular setting is alleviated through learning strong statistical priors from large training datatsets with deep CNNs, which has shown to be successful especially for single shape class settings such as humans. Such approaches also benefit from transfer learning techniques by leveraging networks pretrained on massive general datasets (e.g. ImageNet [6]). While several work [15], [18], [4] show that the learning can be further regularized by integrating differentiable parametric naked human body models (e.g. SMPL [24], GHUM [35], Frank [14]) within deep networks, other methods advocate predicting model-free 3D shape outputs (e.g. [25], [19]), with the prospect of more expressive results, whilst the parametric model is usually involved in generating training 3D pseudoground-truth in this case.

In this work, given a single color image, we tackle the problem of estimating human 3D shape and pose in the form of a fixed-topology triangle mesh, using a feed forward deep neural network. We specifically focus on improving on 978-1-6654-3176-7/21/\$31.00 @2021 European Union a class of model-free methods that propose to use GCNs for this task [19], [9], [20], [21], the graph's vertices and edges being defined as those of the $3 \mathrm{D}$ mesh representing the human shape. Traditionally, these methods extract a global latent feature vector from the image using a $\mathrm{CNN}$, and this same vector is used as input feature to all the mesh vertices equally, as in [19]. The GCN then starts from these mesh-borne features and deforms a T-posed template mesh towards the target posed mesh. A noteworthy variant of this strategy consists in predicting a global feature and mapping it subsequently to initial low resolution mesh vertex features [9], [20], [21], and it was mostly explored for 3D hand prediction rather than full body partly due to the smaller mesh size.

In contrast to these methods, we propose here to use per vertex pixel-aligned local image features as initialization for the GCN stage, as illustrated in Fig.1. We use a method for predicting dense pixel-to-surface correspondences (DensePose [11]) of humans to map each visible vertex in the template geometry to a pixel in the input image. Bi-linear interpolation is then used to build a different feature vector per vertex, by sampling and stacking local image features at the vertex's corresponding 2D location in the image space, at different $\mathrm{CNN}$ feature depths. Given a T-posed initial mesh appended with vertex specific local image features, a GCN regresses the final mesh vertex positions. The network composed of the image CNN and mesh GCN is trained endto-end using $3 \mathrm{D}$ supervision following the training scheme in [25].

We evaluate our method on standard benchmarks for human mesh prediction from images, namely 3DPW [34] and Human3.6M [13]. Our numerical and visual results demonstrate that using local features improves on using only global ones in GCN based human mesh recovery from single image. We also show that our method yields competitive results in comparison to the state-of-the-art methods.

\section{RELATED WORK}

There is a substantial body of work on the subject of human shape and pose estimation from a single image. We review in this section works that we deemed most relevant to the context of our contribution.

\section{A. Optimization-based methods}

Most current optimization-based methods rely on using 2D landmarks such as key-points and silhouettes [29], [3], [10] and optimize parametric models such as SCAPE [2] and SMPL [24] to fit them to these landmarks. The optimization 


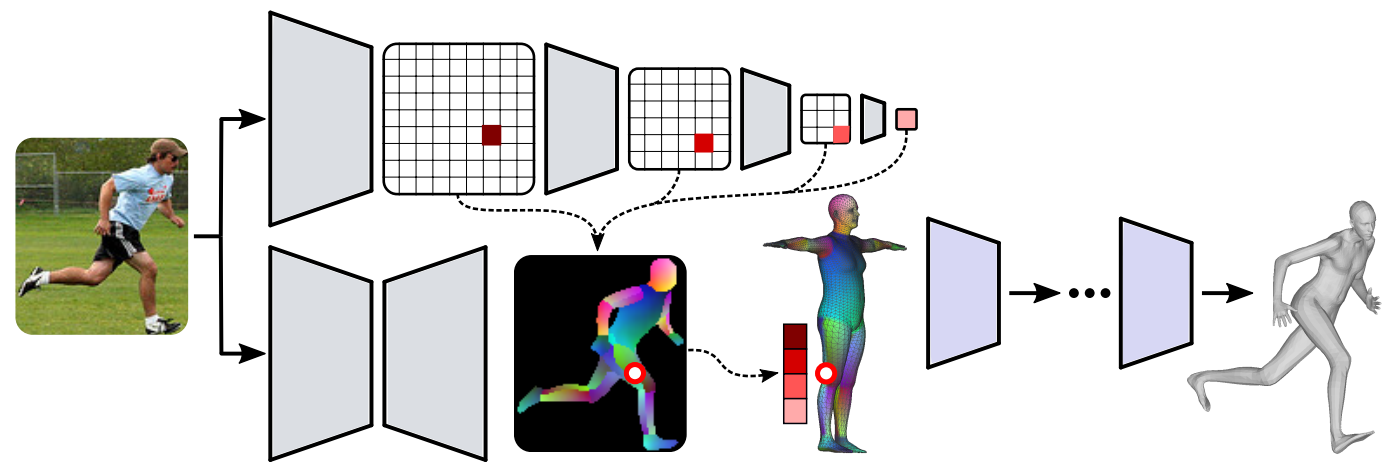

Fig. 1. Overview. Given an input color image, DensePose [11] (Bottom left) produces dense pixel-to-surface correspondences. Meanwhile, an image convolutional neural network (CNN) (Top) builds feature maps at multiple depths (Shades of red). The correspondences are then used to sample (Dashed lines) local image features from the CNN feature maps for each template surface vertex at its corresponding image location (Red Circle). Next, we use a graph convolutional network (GCN) (Right) to map the template surface with vertex specific local image features to the final posed surface.

objective consists of mainly two kinds of loss terms. The first is prior terms designed to penalize unnatural human poses and shapes. The second is data terms minimizing the difference between the inferred 2D landmarks obtained by projecting the predicted mesh onto the image and the groundtruth body landmarks. Additionally, there have been other recent methods [37] which incorporated additional terms such as part segmentation, scene and temporal constraints in the optimization objective. Although optimization-based methods provide generally reliable solutions, they are notoriously slow and prone to getting stuck in local minima especially with challenging initializations. This incentivizes learning-based solutions such as ours, which offer faster inference and do not require initialization.

\section{B. Learning-based methods}

1) Model-based: Model-based methods make use of a parametric 3D human body model to perform 3D human pose and shape estimation. The learning problem is thus reduced to learning the parameters associated with the body model from images and other types of input. A notable example is the work of [15] which directly regresses the SMPL parameters from a single RGB image, by performing a weak supervision comprising of a 2D key-point reprojection error and an adversarially learnt pose prior. SPIN [18] improves on this with a self-improving framework that incorporates 3D human model parameter optimization into network training process. To deal with occlusions and noisy situations which make image-based methods more susceptible to failure, some approaches use alternative inputs such as 2D joint heatmaps [31], silhouettes [27], [32] and semantic segmentation maps [26]. In-spite of the aforementioned advantages behind model-based methods, such strategies can conversely also be somewhat restrictive. The tight relationship between the parameters and the model's output reduces the expressiveness of the generated meshes. Hence, as a model-free method, our framework focuses instead on directly regressing the 3D human body mesh vertices corresponding to an input image following seminal work.

2) Model-free: As the name implies, model-free methods do not rely on parametric models for 3D human body reconstruction. Instead, they directly regress an explicit body shape representation from the input images. Some of the earlier work uses a volumetric reconstruction approach with a voxel output [32], [41]. The main drawback of voxelbased methods is their inability to represent detailed surfaces due to memory limitations. Other methods use different representations such as depth maps, point clouds, etc. [8]. However, these suffer mostly from lacking surface continuity and neighborhood connectivity. To deal with these problems, recent methods propose to directly regress 3D SMPL meshes representing the output human body shape. To the best of our knowledge, this line of work started with CMR [19] which used a GCN to directly regress 3D coordinates of vertices from a global image feature. Pose2Mesh [5] also did the same but from 2D joints as input instead. The work of Lin et al. [22] yields arguably state-of-theart performances through the use of Transformers [33] but requires considerable training time and data. Furthermore, there has been another class of methods focusing on learning dense correspondence between 2D images and 3D shapes. The seminal work of DensePose [11] which provides dense mapping from images to a human body model by regressing 2D correspondence maps has led arguably to the advent of much similar work focusing on dense correspondence. [36], [28] utilized DensePose correspondence maps for 3D human model recovery. However, they only leverage them as input images. Zhang et al. [39] predict and use local and global correspondence maps as input to further $\mathrm{CNN}$ stages for pose and shape prediction in a similar fashion. In contrast, we propose here to use correspondence maps to provide vertices with semantically meaningful image-aligned local features. In fact, our strategy is similar to DecoMR [38], where the authors establish dense correspondences between the surface and the input image, which is subsequently used to transfer image features to the UV-map domain and thereafter perform $3 \mathrm{D}$ coordinate regression with a $2 \mathrm{D} \mathrm{CNN}$. Differently, we explore here the idea of performing vertex position regression from local image features using a convolutional network defined naturally on the same data representation as the output (i.e. GCN on the mesh as opposed to $\mathrm{CNN}$ on the 


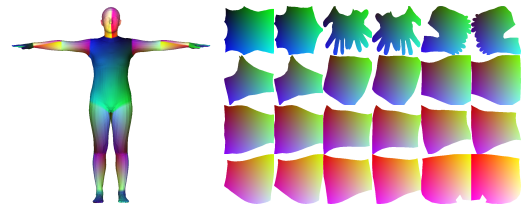

Fig. 2. Illustration of the template geometry IUV mapping.

\section{UV-map).}

\section{METHOD}

We describe in this section the components of our method, which follows the illustration in Fig.1. Our input is a single RGB image and our output is a triangle human mesh with the SMPL [24] template topology. Given the input image, a convolutional neural network (CNN) is tasked with extracting 2D image features. These features are fed to a graph convolutional network $(\mathrm{GCN})$ that transforms a template mesh to the final output mesh. The graph's topology is defined as per that of the mesh. Each vertex in the graph is initialized with a local feature extracted from the image encoder feature maps. This extraction is performed using a DensePose [11] correspondence map that maps pixel locations to the mesh's visible surface.

We use a ResNet50 [12] network that we denote $f$ to build convolutional image feature maps from an input image I, extracted in a coarse-to-fine fashion at 5 network stages:

$$
\left\{\mathrm{F}_{\mathrm{I}}^{l}\right\}_{1 \leq l \leq 5}=f(\mathrm{I}),
$$

$\mathrm{F}_{\mathrm{I}}^{l}$ being the feature map at stage $l$. The feature maps' spatial dimensions decrease gradually from the input image dimensions $(H, W)$ downwards and their respective feature dimensions are 64, 256, 512, 1024 and 2048, summing up to $D=3904$. The final feature is a spatially globally pooled one.

In parallel, the DensePose [11] network, denoted by $h$, predicts a dense mapping from the image pixels to the template mesh, in the form of a 3-channel image IUV:

$$
\mathrm{IUV}=h(\mathrm{I}),
$$

where IUV $\in \llbracket 0,24 \rrbracket \times[0,1] \times[0,1]^{H \times W}$. The first channel indicates which one of 24 pre-defined body parts the pixel belongs to, 0 being the background label. The second and third channels indicate the UV-coordinate of the pixel in a pre-defined UV-map of that body part's template mesh (See Fig.2).

Given a vertex $k$ in the template mesh, the corresponding pixel location $c_{k} \in \llbracket 1, H \rrbracket \times \llbracket 1, W \rrbracket$ in the input image is obtained using the following thresholded nearest-neighbor strategy:

$$
\begin{gathered}
\hat{c}_{k}=\underset{(i, j)}{\arg \min }\left(\left\|\operatorname{IUV}(i, j)-\overline{\mathrm{IUV}}_{k}\right\|_{2}\right), \\
c_{k}= \begin{cases}\hat{c}_{k}, & \text { if }\left\|\operatorname{IUV}\left(\hat{c}_{k}\right)-\overline{\mathrm{IUV}}_{k}\right\|_{2} \leq \delta_{k} \\
\emptyset, & \text { otherwise }\end{cases}
\end{gathered}
$$

where $\overline{\mathrm{IUV}}_{k}$ is the $k^{\text {th }}$ vertex IUV coordinates in the predefined template geometry body partitioning and per part
UV-map (Fig.2). Threshold $\delta_{k}$ is defined as the $k^{\text {th }}$ vertex distance to its closest adjacent neighbor in the template UVmap space. This thresholding insures no pixels are assigned to occluded surface regions.

We construct then the input local mesh-borne feature $\mathrm{F}_{\mathrm{M}}(k)$ for the $k^{\text {th }}$ vertex as follows:

$$
\mathrm{F}_{\mathrm{M}}(k)=\left[\mathrm{F}_{\mathrm{I}}^{1}\left(c_{k}\right), \ldots, \mathrm{F}_{\mathrm{I}}^{4}\left(c_{k}\right), \mathrm{F}_{\mathrm{I}}^{5}, c_{k}, \bar{x}_{k}, \bar{y}_{k}, \bar{z}_{k}\right]
$$

where $\mathrm{F}_{\mathrm{I}}^{l}(\emptyset)=0$ for $1 \leq l \leq 4$ so the non-visible vertices are assigned null local features. We note that the last feature $F_{I}^{5}$ is a global one and hence does not depend on the spatial 2D sampling. $\left(\bar{x}_{k}, \bar{y}_{k}, \bar{z}_{k}\right)$ are the $k^{\text {th }}$ vertex coordinates in the T-pose initial mesh, hence $\mathrm{F}_{\mathrm{M}}(k) \in \mathbb{R}^{D+5}$.

Finally, a GCN $g$, who's topology is defined by the template mesh connectivity, takes as input the mesh local features $\mathrm{F}_{\mathrm{M}} \in \mathbb{R}^{N_{V} \times(D+5)}$ and predicts the final mesh vertex coordinates $\mathrm{M} \in \mathbb{R}^{N_{V} \times 3}$ :

$$
\mathrm{M}=g\left(\mathrm{~F}_{\mathrm{M}}\right),
$$

$N_{V}$ being the total number of vertices. $g$ uses the formulation from [17] and follows the architecture described in [19], where regular graph convolutions are substituted by the semantic graph convolutions introduced in [40]. We note that body joints $\mathbf{J}$ can be obtained from meshes using a fixed linear regressor $W: \mathbf{J}=W \mathrm{M}$.

We train the parameters of the convolutional networks $f$ and $g$ following the losses, training scheme, data augmentation and 3D supervision introduced in [25]. Our loss combines $L 1$ reconstruction errors between the prediction and the ground-truth for mesh vertices $\mathscr{L}_{\text {vertex }}$, joints $\mathscr{L}_{\text {joint }}$, normals $\mathscr{L}_{\text {normal }}$ and edges $\mathscr{L}_{\text {edge }}$ with the following weighting scheme:

$$
\mathscr{L}=\mathscr{L}_{\text {vertex }}+\mathscr{L}_{\text {joint }}+0.1 \mathscr{L}_{\text {normal }}+0.1 \mathscr{L}_{\text {edge }} .
$$

The normal and edge losses are used to ensure smoother and more visually pleasing results.

\section{RESULTS}

We present in this section our experimental setup in addition to our results. We train our network on datasets Human3.6M [13] and MSCOCO [23] following the data augmentation and 3D supervision described in [25]. We use the Adam optimizer [16] in PyTorch on a Quadro RTX 5000 GPU for 12 epochs with a learning rate of $10^{-4}$, followed by another 2 epochs using a learning rate of $10^{-5}$ and finally 1 extra epoch using a learning rate of $10^{-6}$. The image feature extraction network $f$ is initialized with the ImageNet [6] pretrained weights.

We evaluate our contribution numerically using the 3DPW [34] and Human3.6M [13] datasets. We report the MPJPE and PA-MPJPE metrics of our method in Table I when using only one global feature for all vertices $\left(\mathrm{F}_{\mathrm{I}}^{5}\right)$ Global (Ours), and also when using vertex specific local features (full $F_{M}$ ) Local (Ours). For a fair comparison with the competition, we show other methods ([15], [19], [18], [5], [25]) trained 

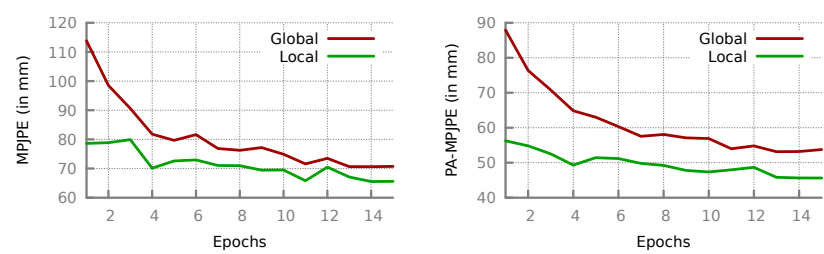

Fig. 3. MPJPE (left) and PA-MPJPE (right) testing losses on Human3.6M [13] for our baseline using only a global image feature (Global) and our proposed approach using per vertex pixel-aligned local features (Local).

\begin{tabular}{c|cc|cc}
\hline Methods & \multicolumn{2}{|c|}{ Human3.6M [13] } & \multicolumn{2}{c}{ 3DPW [34] } \\
& MPJPE & PA-MPJPE & MPJPE & PA-MPJPE \\
\hline HMR [15] & 153.2 & 85.5 & 300.4 & 137.2 \\
GraphCMR [19] & 78.3 & 51.9 & 126.5 & 80.1 \\
SPIN [18] & 72.9 & 85.5 & 113.1 & 71.7 \\
Pose2Mesh [5] & 67.9 & 49.9 & 91.4 & 60.1 \\
I2L-MeshNet [25] & 55.7 & 41.7 & 95.4 & 60.8 \\
\hline Global (Ours) & 70.74 & 53.76 & 112.02 & 67.70 \\
Local (Ours) & 65.61 & 45.62 & 110.31 & 66.52 \\
\hline
\end{tabular}

TABLE I

COMPARISON OF MPJPE AND PA-MPJPE ON HUMAN3.6M [13] AND 3DPW [34]. All Methods are trained on Human3.6M [13] AND MSCOCO [23].

on the same data as us and we relay their performances as they were reported in [25].

We firstly showcase the effect of using local features on the convergence of our model in Fig. 3. For both of the PAMPJPE and MPJPE metrics, our pixel-aligned local mesh features enable the model to reach significantly lower generalization errors on Human3.6M [13] at the same training epoch compared to our global feature baseline. Furthermore, we note that our Global baseline shares the same network design as GraphCMR [19]. However, by substituting regular convolutions with learnable adjacency matrix ones [40] and training with the same supervision and training scheme as in [25], we manage to improve its performance by roughly $8 \mathrm{~mm}$ in MPJPE for Human3.6M [13], 14mm in MPJPE and $13 \mathrm{~mm}$ in PA-MPJPE for 3DPW [34]. Our proposed Local method improves on our Global baseline substantially in almost all figures, by roughly $5 \mathrm{~mm}$ in MPJPE and $8 \mathrm{~mm}$ in PA-MPJPE for Human3.6M [13], and 2mm in MPJPE for 3DPW [34]. It is noteworthy that our Local version also achieves competitive results in comparison to the state-ofthe-art, as it outperforms all methods presented in the table on Human3.6M [13] except for I2L-MeshNet [25], while ranking $3^{\text {rd }}$ on 3DPW [34] closely behind I2L-MeshNet [25] and Pose2Mesh [5], which uses 2D joints (from HRNet [30]) as input rather than a RGB image. While [5] is advantaged by the $2 \mathrm{D}$ pose input, we believe the performance of [25] is by virtue of their Lixel architecture which is not readily applicable to irregular graphs.

The numerical superiority of our contribution compared to our baseline is also confirmed with qualitative results. As shown in Fig.4, we notice that using local image features in the GCN stage yields improved visual results, as witnessed by these examples from the 3DPW [34] and Human3.6M [13] datasets. The red boxes in the figure illustrate in

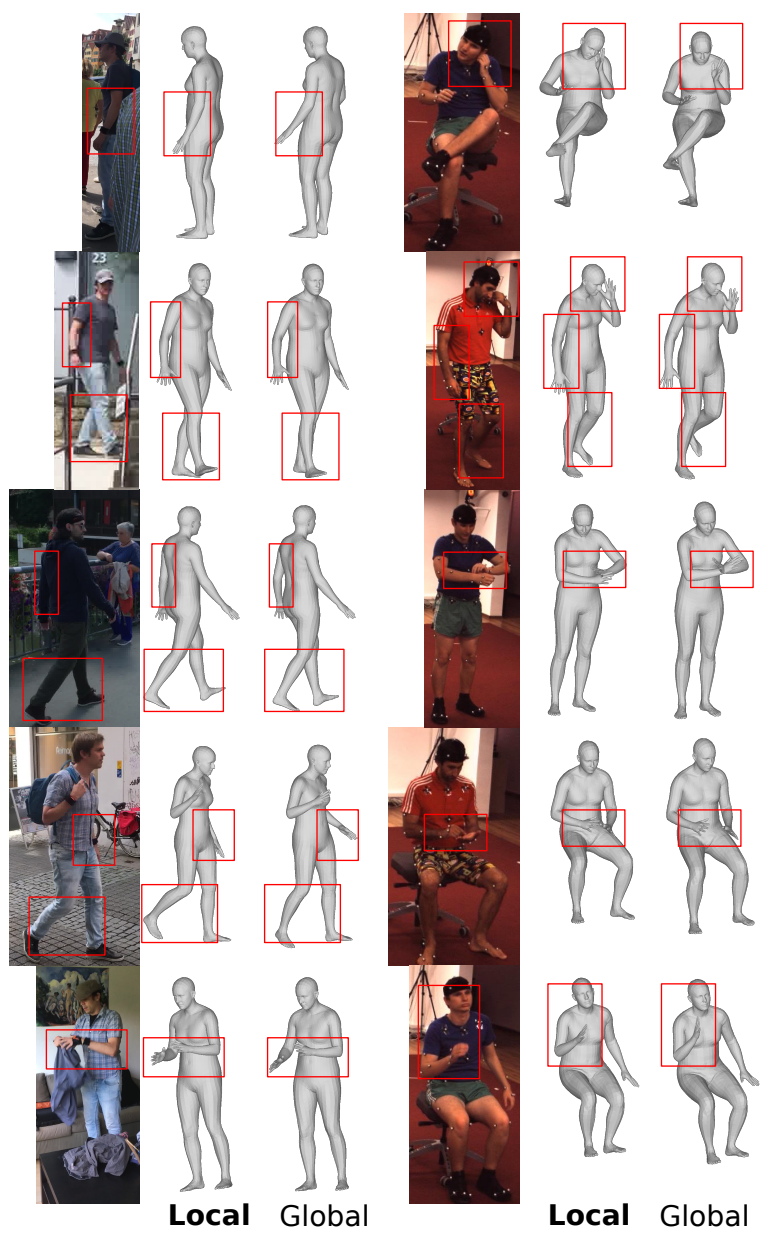

Fig. 4. Comparison of our baseline using only a global image feature (Global) and our proposed approach using per vertex pixel-aligned local features (Local) on the 3DPW [34] (left) and Human3.6M [13] (right) datasets.

particular the better positioning of the body limbs with our Local method compared to our Global baseline. We note that following [19], we show the results after an additional linear layer trained to predict SMPL instances from the previous output for smoother visual results.

\section{CONCLUSION}

We presented a method for 3D human shape and pose estimation from a single RGB image. The method is modelfree and relies on a GCN that starts from a template T-posed mesh and regresses the final vertex coordinates. Contrarily to seminal work [19], we propose to initialize the graph convolutions with pixel-aligned vertex-specific features instead of only one global feature. These features are extracted at multiple feature map stages of an image $\mathrm{CNN}$, and mapped subsequently to the graph vertices using a pixel-tosurface correspondence map [11]. Our results demonstrated the benefit of using local features in GCN based human 3D shape and pose estimation. Next, we will attempt to make the entire pipeline fully differentiable, by including the correspondence estimation network $h$ training in the end-toend learning framework alongside the image feature network $f$ and graph network $g$. 


\section{REFERENCES}

[1] V. F. Abrevaya, A. Boukhayma, P. H. Torr, and E. Boyer. Crossmodal deep face normals with deactivable skip connections. In Proceedings of the IEEE/CVF Conference on Computer Vision and Pattern Recognition, pages 4979-4989, 2020.

[2] D. Anguelov, P. Srinivasan, D. Koller, S. Thrun, J. Rodgers, and J. Davis. Scape: shape completion and animation of people. In $A C M$ SIGGRAPH 2005 Papers, pages 408-416. 2005.

[3] F. Bogo, A. Kanazawa, C. Lassner, P. Gehler, J. Romero, and M. J. Black. Keep it smpl: Automatic estimation of $3 \mathrm{~d}$ human pose and shape from a single image. In European conference on computer vision, pages 561-578. Springer, 2016.

[4] A. Boukhayma, R. d. Bem, and P. H. Torr. 3d hand shape and pose from images in the wild. In Proceedings of the IEEE/CVF Conference on Computer Vision and Pattern Recognition, pages 10843-10852, 2019.

[5] H. Choi, G. Moon, and K. M. Lee. Pose2mesh: Graph convolutional network for $3 \mathrm{~d}$ human pose and mesh recovery from a $2 \mathrm{~d}$ human pose. In European Conference on Computer Vision, pages 769-787. Springer, 2020.

[6] J. Deng, W. Dong, R. Socher, L.-J. Li, K. Li, and L. Fei-Fei. Imagenet: A large-scale hierarchical image database. In 2009 IEEE conference on computer vision and pattern recognition, pages 248-255. Ieee, 2009.

[7] Y. Feng, F. Wu, X. Shao, Y. Wang, and X. Zhou. Joint 3d face reconstruction and dense alignment with position map regression network. In Proceedings of the European Conference on Computer Vision (ECCV), pages 534-551, 2018.

[8] V. Gabeur, J.-S. Franco, X. Martin, C. Schmid, and G. Rogez. Moulding humans: Non-parametric $3 \mathrm{~d}$ human shape estimation from single images. In Proceedings of the IEEE/CVF International Conference on Computer Vision, pages 2232-2241, 2019.

[9] L. Ge, Z. Ren, Y. Li, Z. Xue, Y. Wang, J. Cai, and J. Yuan. $3 \mathrm{~d}$ hand shape and pose estimation from a single rgb image. In Proceedings of the IEEE/CVF Conference on Computer Vision and Pattern Recognition, pages 10833-10842, 2019.

[10] P. Guan, A. Weiss, A. O. Balan, and M. J. Black. Estimating human shape and pose from a single image. In 2009 IEEE 12th International Conference on Computer Vision, pages 1381-1388. IEEE, 2009.

[11] R. A. Güler, N. Neverova, and I. Kokkinos. Densepose: Dense human pose estimation in the wild. In Proceedings of the IEEE conference on computer vision and pattern recognition, pages 7297-7306, 2018.

[12] K. He, X. Zhang, S. Ren, and J. Sun. Deep residual learning for image recognition. In Proceedings of the IEEE conference on computer vision and pattern recognition, pages 770-778, 2016.

[13] C. Ionescu, D. Papava, V. Olaru, and C. Sminchisescu. Human3. 6m: Large scale datasets and predictive methods for $3 \mathrm{~d}$ human sensing in natural environments. IEEE transactions on pattern analysis and machine intelligence, 36(7):1325-1339, 2013.

[14] H. Joo, T. Simon, and Y. Sheikh. Total capture: A 3d deformation model for tracking faces, hands, and bodies. In Proceedings of the IEEE conference on computer vision and pattern recognition, pages $8320-8329,2018$

[15] A. Kanazawa, M. J. Black, D. W. Jacobs, and J. Malik. End-toend recovery of human shape and pose. In Proceedings of the IEEE conference on computer vision and pattern recognition, pages 7122 7131, 2018

[16] D. P. Kingma and J. Ba. Adam: A method for stochastic optimization. arXiv preprint arXiv:1412.6980, 2014

[17] T. N. Kipf and M. Welling. Semi-supervised classification with graph convolutional networks. arXiv preprint arXiv:1609.02907, 2016.

[18] N. Kolotouros, G. Pavlakos, M. J. Black, and K. Daniilidis. Learning to reconstruct $3 \mathrm{~d}$ human pose and shape via model-fitting in the loop. In Proceedings of the IEEE/CVF International Conference on Computer Vision, pages 2252-2261, 2019.

[19] N. Kolotouros, G. Pavlakos, and K. Daniilidis. Convolutional mesh regression for single-image human shape reconstruction. In Proceedings of the IEEE/CVF Conference on Computer Vision and Pattern Recognition, pages 4501-4510, 2019.

[20] D. Kulon, R. A. Guler, I. Kokkinos, M. M. Bronstein, and S. Zafeiriou. Weakly-supervised mesh-convolutional hand reconstruction in the wild. In Proceedings of the IEEE/CVF Conference on Computer Vision and Pattern Recognition, pages 4990-5000, 2020.

[21] D. Kulon, H. Wang, R. A. Güler, M. Bronstein, and S. Zafeiriou. Single image $3 \mathrm{~d}$ hand reconstruction with mesh convolutions. arXiv preprint arXiv:1905.01326, 2019.

[22] K. Lin, L. Wang, and Z. Liu. End-to-end human pose and mesh reconstruction with transformers. In Proceedings of the IEEE/CVF
Conference on Computer Vision and Pattern Recognition, pages 1954 1963, 2021

[23] T.-Y. Lin, M. Maire, S. Belongie, J. Hays, P. Perona, D. Ramanan, P. Dollár, and C. L. Zitnick. Microsoft coco: Common objects in context. In European conference on computer vision, pages 740-755. Springer, 2014.

[24] M. Loper, N. Mahmood, J. Romero, G. Pons-Moll, and M. J. Black. Smpl: A skinned multi-person linear model. ACM transactions on graphics (TOG), 34(6):1-16, 2015.

25] G. Moon and K. M. Lee. I21-meshnet: Image-to-lixel prediction network for accurate $3 \mathrm{~d}$ human pose and mesh estimation from a single rgb image. In Computer Vision-ECCV 2020: 16th European Conference, Glasgow, UK, August 23-28, 2020, Proceedings, Part VII 16, pages 752-768. Springer, 2020.

[26] M. Omran, C. Lassner, G. Pons-Moll, P. Gehler, and B. Schiele. Neural body fitting: Unifying deep learning and model based human pose and shape estimation. In 2018 international conference on $3 D$ vision $(3 D V)$, pages 484-494. IEEE, 2018.

[27] G. Pavlakos, L. Zhu, X. Zhou, and K. Daniilidis. Learning to estimate $3 \mathrm{~d}$ human pose and shape from a single color image. In Proceedings of the IEEE Conference on Computer Vision and Pattern Recognition, pages 459-468, 2018

[28] Y. Rong, Z. Liu, C. Li, K. Cao, and C. C. Loy. Delving deep into hybrid annotations for $3 \mathrm{~d}$ human recovery in the wild. In Proceedings of the IEEE/CVF International Conference on Computer Vision, pages 5340-5348, 2019.

[29] L. Sigal, A. Balan, and M. Black. Combined discriminative and generative articulated pose and non-rigid shape estimation. Advances in neural information processing systems, 20:1337-1344, 2007.

[30] K. Sun, B. Xiao, D. Liu, and J. Wang. Deep high-resolution representation learning for human pose estimation. In Proceedings of the IEEE/CVF Conference on Computer Vision and Pattern Recognition, pages 5693-5703, 2019

[31] H.-Y. Tung, H.-W. Tung, E. Yumer, and K. Fragkiadaki. Selfsupervised learning of motion capture. In I. Guyon, U. V. Luxburg, S. Bengio, H. Wallach, R. Fergus, S. Vishwanathan, and R. Garnett, editors, Advances in Neural Information Processing Systems 30, pages 5236-5246. 2017

[32] G. Varol, D. Ceylan, B. Russell, J. Yang, E. Yumer, I. Laptev, and C. Schmid. Bodynet: Volumetric inference of $3 \mathrm{~d}$ human body shapes. In Proceedings of the European Conference on Computer Vision (ECCV), pages 20-36, 2018.

[33] A. Vaswani, N. Shazeer, N. Parmar, J. Uszkoreit, L. Jones, A. N. Gomez, Ł. Kaiser, and I. Polosukhin. Attention is all you need. In Advances in neural information processing systems, pages 5998-6008, 2017.

[34] T. von Marcard, R. Henschel, M. J. Black, B. Rosenhahn, and G. PonsMoll. Recovering accurate $3 \mathrm{~d}$ human pose in the wild using imus and a moving camera. In Proceedings of the European Conference on Computer Vision (ECCV), pages 601-617, 2018.

[35] H. Xu, E. G. Bazavan, A. Zanfir, W. T. Freeman, R. Sukthankar, and C. Sminchisescu. Ghum \& ghuml: Generative $3 \mathrm{~d}$ human shape and articulated pose models. In Proceedings of the IEEE/CVF Conference on Computer Vision and Pattern Recognition, pages 6184-6193, 2020.

[36] Y. Xu, S.-C. Zhu, and T. Tung. Denserac: Joint 3d pose and shape estimation by dense render-and-compare. In Proceedings of the IEEE/CVF International Conference on Computer Vision, pages 7760 $7770,2019$.

[37] A. Zanfir, E. Marinoiu, and C. Sminchisescu. Monocular 3d pose and shape estimation of multiple people in natural scenes-the importance of multiple scene constraints. In Proceedings of the IEEE Conference on Computer Vision and Pattern Recognition, pages 2148-2157, 2018.

[38] W. Zeng, W. Ouyang, P. Luo, W. Liu, and X. Wang. 3d human mesh regression with dense correspondence. In Proceedings of the IEEE/CVF Conference on Computer Vision and Pattern Recognition, pages 7054-7063, 2020.

[39] H. Zhang, J. Cao, G. Lu, W. Ouyang, and Z. Sun. Learning 3d human shape and pose from dense body parts. IEEE Transactions on Pattern Analysis and Machine Intelligence, 2020

[40] L. Zhao, X. Peng, Y. Tian, M. Kapadia, and D. N. Metaxas. Semantic graph convolutional networks for $3 \mathrm{~d}$ human pose regression. In Proceedings of the IEEE/CVF Conference on Computer Vision and Pattern Recognition, pages 3425-3435, 2019.

[41] Z. Zheng, T. Yu, Y. Wei, Q. Dai, and Y. Liu. Deephuman: 3d human reconstruction from a single image. In Proceedings of the IEEE/CVF International Conference on Computer Vision, pages 77397749, 2019 